\title{
A Single Nucleotide Change in the Prolidase Gene in Fibroblasts from Two Patients with Polypeptide Positive Prolidase Deficiency Expression of the Mutant Enzyme in NIH 3T3 Cells
}

Akito Tanoue, Fumio Endo, Akito Kitano, and Ichiro Matsuda

Department of Pediatrics, Kumamoto University Medical School, Kumamoto 860, Japan

\begin{abstract}
Prolidase deficiency is an autosomal recessive disorder characterized by mental retardation and various skin lesions. Cultured skin fibroblasts were obtained from two independent patients with abnormal prolidase. Using the polymerase chain reaction, we amplified the entire coding region of human prolidase mRNA derived from patients' fibroblasts. Nucleotide sequence analysis of amplified CDNA products revealed a $G$ to $A$ substitution at position 826 in exon 12, where aspartic acid was replaced by asparagine at the amino acid residue 276 , in cells from both patients. An analysis of the DNA showed that the substitution was homozygous.
\end{abstract}

An expression plasmid clone containing a normal human prolidase cDNA (pEPD-W) or mutant prolidase cDNA (pEPD-M) was prepared, transfected, and tested for expression in NIH 3T3 cells. Incorporation of pEPD-W and pEPD$M$ resulted in the synthesis of an immunological polypeptide that corresponded to human prolidase. Active human enzyme was detected in cells transfected with pEPD-W, but not in those transfected with pEPD-M. These results were compatible with our observation of fibroblasts and confirmed that the substitution was responsible for the enzyme deficiency.

As active prolidase was recovered in prolidase-deficient fibroblasts transfected with pEPD-W, this restoration of prolidase activity after transfection means that gene replacement therapy for individuals with this human disorder can be given due consideration. (J. Clin. Invest. 1990. 86:351-355.) Key words: prolidase $\bullet$ prolidase deficiency $\bullet$ polymerase chain reaction • point mutation • transfection

\section{Introduction}

Prolidase is a homodimeric enzyme catalyzing the hydrolysis of dipeptides with carboxy-terminal proline. This enzyme

Part of this work was presented in Japanese at the Annual Meeting of the Japanese Society for Inborn Errors of Metabolism, Fukui, Japan, October 1989.

Please address reprint requests to Dr. Fumio Endo, Department of Pediatrics, Kumamoto University Medical School, Honjo 1-1-1, Kumamoto 860, Japan.

Received for publication 14 February 1990 and in revised form 9 April 1990.

J. Clin. Invest.

(c) The American Society for Clinical Investigation, Inc.

0021-9738/90/07/035i/05 $\$ 2.00$

Volume 86, July 1990, 351-355 seems to play an important role in the recycling of proline. Prolidase deficiency is an autosomal recessive disorder with a variable clinical phenotype and a metabolic phenotype, notably massive imidodipeptiduria (1). Among patients with clinical symptoms of this disorder, skin ulcers, mental retardation, and abnormalities in bones occur. Data on 28 patients with prolidase deficiency have been documented in the past 20 years (1), and we $(2,3)$ and others $(4)$ have shown that there were at least three types of mutations: immunologically crossreacting material (CRM) ${ }^{1}$ negative, normal-size CRM, and enlarged CRM.

We identified a cDNA clone corresponding to the human prolidase (5), and determined the nucleotide sequence and the primary structure of the enzyme (6). The prolidase gene is located on chromosome $19(6)$ and consists of 15 exons and 14 introns, the span being $>130 \mathrm{~kb}(2 \mathrm{a})$. We have now examined the molecular events in two patients with polypeptide positive prolidase deficiency. The nucleotide sequences of cDNAs from the patients were analyzed by making comparisons with the sequence of cDNA and genomic DNA from a normal individual. We obtained evidence for the same amino acid substitution in the mutant enzymes, thereby confirming that this substitution is responsible for defects in the activity.

\section{Methods}

Materials. Purified human prolidase, a monoclonal antibody (EP2) directed to human prolidase, and antiserum raised against human prolidase were prepared as described (2). [ $\left.\alpha-{ }^{32} \mathrm{P}\right] \mathrm{dCTP}(3,000 \mathrm{Ci} /$ mmol) was purchased from ICN Radiochemicals (Irvine, CA). Nitrocellulose membrane was obtained from Schleicher and Schuell (Dassel, FRG). Restriction enzymes, T4 DNA ligase, and Taq DNA polymerase were purchased from Takara Shuzo Co. (Kyoto, Japan) and were used essentially as recommended by the supplier. Avian myeloblastosis virus reverse transcriptase and the reagent lipofectin were purchased from Bethesda Research Laboratories (Gaithersburg, MD). A 7-deaza sequencing kit was obtained from Toyobo Co. (Osaka, Japan). Oligonucleotide primers were synthesized using a DNA synthesizer (model 381 A; Applied Biosystems Inc., Foster City, CA).

Polymerase chain reaction of $m R N A$. Total cellular RNAs from controls' and patients' fibroblasts were prepared using the guanidium thiocyanate procedure (7). Five oligonucleotide primers were synthesized for polymerase chain reaction (PCR) of $\mathrm{mRNA}$, an oligonucleotide primer C, and two sets of oligonucleotide primers (A1, A2, B1, B2) consisting of 20 bases complementary to the published normal sequence of human prolidase (6) (Fig. 1).

1. Abbreviations used in this paper: $\mathrm{CRM}$, crossreacting material; $\mathrm{PCR}$, polymerase chain reaction. 


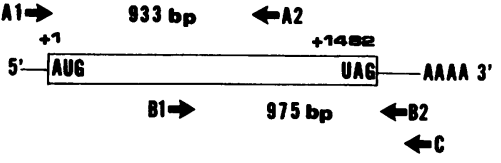

Primer set A

Al; 5 ' CCGGTGCCGgGCGAACATGG 3 '

A2; 5 ' CACCAGTCACCTGGCTTCAT 3 '

Primer set $B$

Bl; 5 ' TTGGAAAGCCTCTTCGAGCA3 '

B2; 5' CCCGGGAAACAGCACTGTTT3'

Primer $C$

$$
5 \text { 'GTGGTGCCTGCAAAAggGTA3' }
$$

Figure 1. Strategy for PCR amplification and the DNA sequence of oligonucleotide primers. The PCR primers Al, $A 2, B 1$, and $B 2$ were used to amplify prolidase sequences from specific primer $C$ primed DNA:RNA hybrids. The relative priming sites and direc-

tion of extension for each primer (arrows) are indicated. The bottom panel shows the DNA sequence of PCR oligonucleotide primers; A1 (-16 to +4$),$ A2 (958 to 977), B1 (664 to 683), B2 (1,620 to 1,639), and $C(1,659$ to 1,678$)$ are complementary to the sequence of prolidase (6). Of these, A2, B2, and C represent reverse or antisense sequences. Two segments, 933- and 975-bp long, were amplified with primer sets of A and B, respectively. The base number for the A of the ATG start codon and the terminal G of the UAG stop codon are indicated.

A specific first-strand cDNA copy of human prolidase RNA was made from $10 \mu \mathrm{g}$ of total cellular RNA using the oligonucleotide primer $\mathrm{C}$ complementary to the RNA strand that serves as a primer for reverse transcriptase (8). Amplification of the first strand of cDNA was carried out according to the method of Saiki et al. (9) using Taq DNA polymerase. 30 cycles of PCR amplification were performed with a DNA thermal cycler (Perkin-Elmer Cetus Corp., Hayward, CA). Each cycle ran for $1 \mathrm{~min}$ at $94^{\circ} \mathrm{C}$ to denature the double-stranded DNA, 2 min at $55^{\circ} \mathrm{C}$ for the primers to anneal to their complementary sequences, and 5 min at $72^{\circ} \mathrm{C}$ for extension of the DNA strands.

Analysis of amplified cDNA. Specific amplified cDNA fragments of the human prolidase were ligated into the plasmid vector pUC18 with T4 DNA ligase. Several independent clones obtained from each PCR amplification were sequenced according to the dideoxy-chain termination method (10), using synthetic oligonucleotide primers complementary to either the sequence of the vector or the prolidase cDNA sequence.

Amplification and analysis of genomic DNA. Human nuclear DNA was prepared from fibroblasts and lymphoblast cells with prolidase deficiency (3), or peripheral leukocytes of normal individuals, as described by Kunkel et al. (11). Approximately $1 \mu \mathrm{g}$ of DNA was amplified with the use of a set of oligonucleotide primers (Fig. 3) complementary to the normal sequence of the human prolidase gene. The $5^{\prime}$ primer ( 24 mer) corresponded to the sequence in intron 11 and the $3^{\prime}$ primer ( 25 mer) to the sequence in intron 12 (2a). PCR was performed as described above, except for $2 \mathrm{~min}$ at $72^{\circ} \mathrm{C}$ for the extension. The amplified DNA fragment was digested with a restriction enzyme of a Taq I and analyzed on a $2 \%$ agarose gel.

Plasmid construction. A wild type of cDNA clone (pPD-1) covering the entire coding region of human prolidase was constructed by PCR of the mRNA from normal human fibroblasts. A wild type of expression clone (pEPD-W) was constructed as follows: the 1.7-kb Eco RI fragment derived from pPD-1 was isolated and inserted into an expression vector pCAGGS, a derivative of pAGS-3 (12), which has the cytomegalovirus intermediate early enhancer, the chicken $\beta$-actin promoter, and a polyadenylation signal of the rabbit $\beta$-globin gene. A mutant type of expression clone (pEPD-M) was produced by substituting the 154-bp Sac I fragment (784-937) of human prolidase cDNA insert in pEPD-W with the 154-bp Sac I fragment (784-937) with a point mutation. The Sac I fragment was derived from a subclone of the patient's $\mathrm{CDNA}$, which included the sequence of the putative mutation (position 826). The nucleotide sequence and a proper $5^{\prime}$ to $3^{\prime}$ orientation of the prolidase cDNA in each clone was confirmed by restriction digestion, gel analysis, and sequence. The plasmids grown in Esche- richia coli HB101 were purified by two cycles of cesium chlorideethidium bromide centrifugation.

Cells and DNA transfection. Cultured NIH 3T 3 cells and fibroblasts with prolidase deficiency were used for transfection. Transfection of DNA was performed using lipofection (13), according to instructions given by the supplier. Nearly confluent cells $(80 \%)$ in $75-\mathrm{cm}$ dishes were washed twice with $6 \mathrm{ml}$ of RPMI 1640 medium (Gibco Laboratories, Grand Island, NY), and $9 \mathrm{ml}$ of RPMI 1640 medium containing $10 \mu \mathrm{g}$ of DNA and $30 \mu \mathrm{g}$ of lipofection was added. To normalize for any variability in the transfection efficiency, plasmid pCH110 which contained the $E$. coli lac $\mathrm{Z}$ gene sequence under the control of Simian virus 40 enhancer-promoter (14), was cotransfected as an internal standard. The transfected cells were incubated for $6 \mathrm{~h}$ in a $7 \% \mathrm{CO}_{2}$ atmosphere at $37^{\circ} \mathrm{C}$, after which $9 \mathrm{ml}$ of RPMI 1640 medium with $20 \%$ FCS (Gibco Laboratories) was added. After incubation of this preparation for $12 \mathrm{~h}$, the medium was replaced with $18 \mathrm{ml}$ of fresh medium with $10 \%$ FCS and the cells were harvested $2 \mathrm{~d}$ later.

Cell extracts were prepared as described (3) and used for protein assay, immune precipitation, and immune blot analyses. Part of the cell extracts were assayed for $\beta$-galactosidase activity by incubation with $O$-nitrophenyl- $\beta$-D-galactopyranoside as described (15). The amount of protein was measured by a dye-binding method (kit from Bio-Rad Laboratories, Richmond, CA).

Enzyme assay, immune precipitation, and immune blot. Prolidase activities in the crude cell extracts were measured as described (16). Immune precipitation of human prolidase with the mouse monoclonal antibody (EP2) was carried out as described (2). The prolidase activity in the immune complex was measured as described (3) and conditions for immune blots were also as described $(2,3)$.

\section{Results}

Human prolidase is composed of 492 amino acid residues synthesized from the 1,482-base coding region of the mRNA sequence (6). Although the PCR technique has been used to isolate DNA fragments as large as $2,000 \mathrm{bp}$, the efficiency of the technique is improved when target sequences of $<1,000$ bp are used (17). As shown in Fig. 1, we used primer sets of $A$ and $B$ to amplify 933- and 975-bp segments, respectively. Together, these overlapping segments covered the entire coding region of human prolidase mRNA.

Two fibroblast strains from two independent patients with polypeptide positive prolidase deficiency were analyzed. $\mathrm{Pa}$ tient 1 (female) (18) died at age 36, and patient 2 (male) (19) was 8 yr old. Both patients presented with skin lesions. These two families are apparently unrelated; however, both families came from the Middle East. Using primer sets, specific prolidase cDNA fragments were successfully amplified from total cellular RNA from the control or the patients. Each amplified DNA was the same size, as compared with findings in the control, thereby suggesting that there were no deletions or insertions in mRNA in the patients' fibroblasts.

Sequencing of the amplified cDNA from these patients revealed a single-base substitution ( $G$ to $A$ at position 826 ) that resulted in the replacement of Asp with Asn at amino acid residue 276 in exon 12 (Figs. 2 and 3). This replacement was detected in all five independent subclones after amplification from the fibroblasts of patient 1 and four subclones from patient 2 with the primer set of $A$ and B. To confirm that the substitution was not due to PCR or to a cloning artifact, and was homozygous, we analyzed genomic DNA from the patients' fibroblasts. The nucleotide sequence, including exon 12, was amplified with the primer set as shown in Fig. 3. 


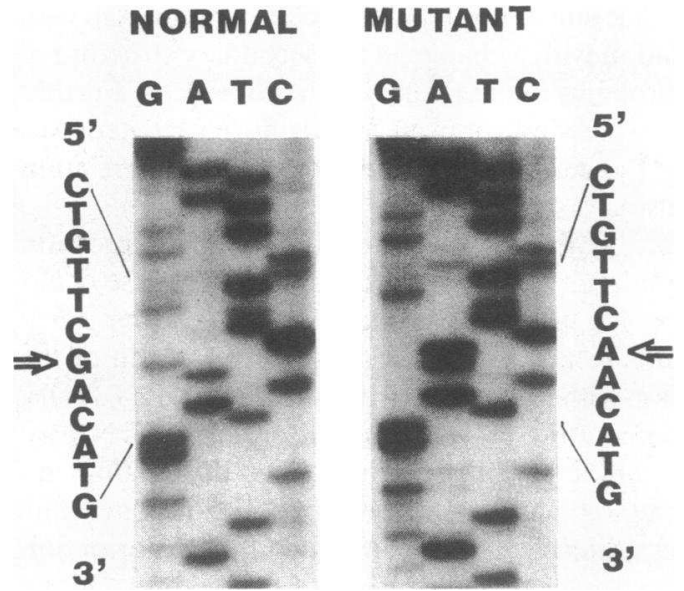

Figure 2. Partial nucleotide sequences of the normal and mutated prolidase cDNA. The autoradiographs of the nucleotide sequence of the mutated region are shown. The sequence of mutated cDNA from patient 1 is compared with that of the control. The single base substitution of $A$ for $G$ at position 826 is indicated by an arrow.

Using this primer set, a 222-bp fragment was amplified in the case of the control (Fig. 3, lane $N$ ), patient 1 (lane $P 6$ ), and patient 2 (lane $P 7$ ). When amplified DNA from the control was digested with Taq I, 171- and 51-bp fragments were generated (lane $N$ ). On the other hand, the 222-bp fragments from patients 1 and 2 (Fig. 3, lanes $P 6$ and $P 7$ ) remained unchanged after digestion with Taq $I$. The $G$ to $A$ substitution at position 826 in exon 12 resulted in the elimination of a Taq I site, which cleaves at the $5^{\prime}$-TCGA-3' but not the 5'-TCAA-3' sequence. This result suggests that these patients have a homozygous allele and that the substitution was not due to a PCR artifact of the mRNA. In other patients with a prolidase deficiency there was a lack of prolidase activity and CRM (3), and the pattern was similar (lanes $P 1-P 5$ ) to the control, hence the probability of a different mutation(s).

An analysis of transfection and expression was made to determine if the substitution was indeed responsible for the prolidase deficiency in these patients. We constructed a pEPD-W with a normal sequence of human prolidase and a pEPD-M with a $G$ to A substitution at position 826 . The results are shown in Fig. 4. Endogenous prolidase in the NIH 3T3 cell was present (data not shown), but only human prolidase immune-precipitated with EP2. An immunological protein of $56 \mathrm{kD}$ crossreactive with the anti-human prolidase antibody is present in cells transfected with pEPD-M or pEPD-W (Fig. $4 A$, lanes 3 and 4 ) but not in those transfected with pCAGGS (lane 2). Prolidase activity was detected in cells transfected with pEPD-W and corresponding to $60 \%$ of the normal human fibroblasts (Fig. $4 \mathrm{~B}$ ), whereas prolidase activity was either absent or markedly reduced in cells transfected with pCAGGS or pEPD-M. Thus, the cells transfected with pEPD-M could synthesize immunological polypeptide, but the catalytic activity was severely impaired.

5' TTGGATCCCCAGTACCCACTGCCC

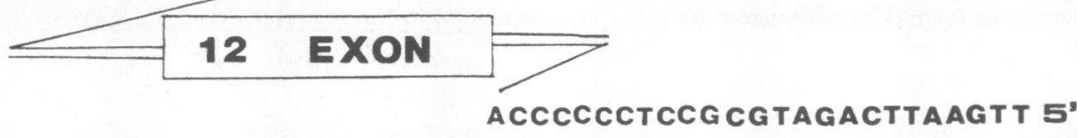

Figure 3. Analysis of prolidase genotype in exon 12 by restriction endonuclease Taq I digestion. The 5'-oligonucleotide primer was a 24-mer ( $5^{\prime}$ TTGGATCCCCAGTACCCACTGCCC3') that contained 20 bases of intron 11 sequence. The 3'-oligonucleotide primer was a $25-\operatorname{mer}(5$ TTGAATTCAGAT-

Mutant

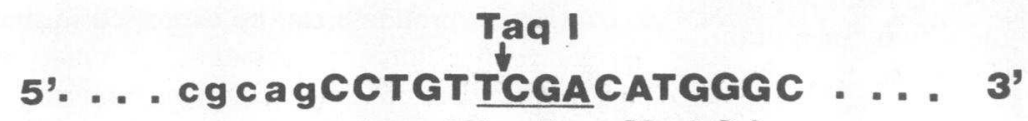
LeuPheAs pMe tG Iy GCGCCTCCCCCCA3') that contained 20 bases of intron 12 sequence. Middle panel shows the nucleotide and amino acid sequences in exon 12. Exon sequence is shown in capital letters and intron sequences are in lowercase letters. A 222-bp fragment was amplified from genomic DNA sequence from the control and seven prolidase-deficient patients. The resulting PCR products, both before (-) and after $(+)$ digestion with a Taq I were analyzed on a $2 \%$ agarose gel. The gel was stained with ethidium bromide. DNAs amplified from the mutant homozygotes (lanes $P 6$ and $P 7$ ) contain the sequence $5^{\prime}-$ TCAA-3' (underlined) and are not cleaved by the enzyme. DNAs derived from the control (lane $N$ ) and other patients (lanes $P 1-P 5$ ) contain the sequence $5^{\prime}$-TCGA-3' and are cleaved by a Taq I (arrow). Lane $M$ shows molecular size marker. 

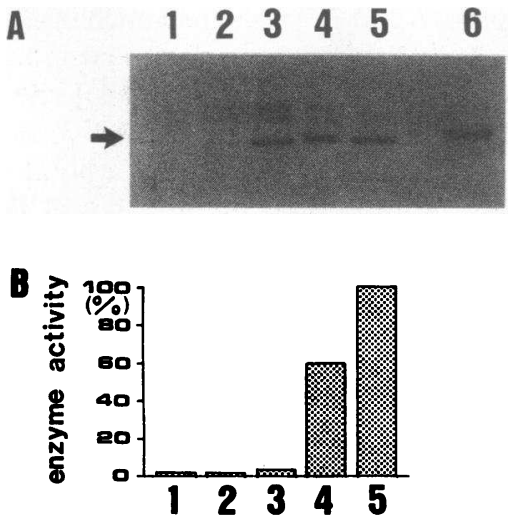

Figure 4. Immune blot analysis $(A)$ and relative enzyme activities $(B)$ of human prolidase from transfected NIH 3T3 cells. pCH110 was used as the negative control for background activity, and human fibroblasts were used as positive control for prolidase activity. NIH 3T3 cells were transfected with $10 \mu \mathrm{g}$ prolidase cDNA and/or $5 \mu \mathrm{g}$ pCH 110 cDNA using $30 \mu \mathrm{g}$ lipo-

fection, then harvested on the third day of transient expression. $(A)$, Proteins from NIH 3T3 cells transfected with pCH110 (lane 1), pCH110+pCAGGS (lane 2), pCH110+pEPD-M (lane 3), and pCH110+pEPD-W (lane 4) were subjected to immune precipitation. Immune precipitates were separated on SDS-PAGE (20) and analyzed by immune blot as described in Methods. $100 \mu \mathrm{g}$ of cell extracts was used for immune precipitation. Proteins from a normal human fibroblast (lane 5) and purified prolidase protein from human erythrocytes (lane 6) were analyzed for comparison. An arrow indicates the $56-\mathrm{kD}$ protein of human prolidase. $(B)$, Relative prolidase activities expressed in the transfected NIH $3 \mathrm{~T} 3$ cells $(1, \mathrm{pCH} 110 ; 2$, pCH1 10+pCAGGS; 3, pCH1 10+pEPD-M; 4, pCH110+pEPD-W) and in the control fibroblasts (5). The immune-precipitated activities were calculated based on the amount of protein in the cell extracts and expressed by the percentage of that of control human fibroblast. The activities of $\beta$-galactosidase in the cells transfected with pCH110 were measured to monitor the efficiency of transfection. Since $\beta$-galactosidase activities were essentially similar in the transfected cells (not shown), prolidase activity was not formally normalized for transfection efficiency.

When pEPD-W transfected into fibroblasts from patients, the prolidase activity corresponding to the activity seen in the normal human fibroblasts was recovered (Fig. 5).

\section{Discussion}

Mutations can lead to decreased functions of prolidase, resulting in the clinical and biochemical phenotype of a prolidase deficiency. We obtained evidence that the $G$ to A substitution in a region of the prolidase gene, which was homozygous in these cells, resulted in a marked decrease in prolidase activity, hence a phenotype of polypeptide positive prolidase deficiency.

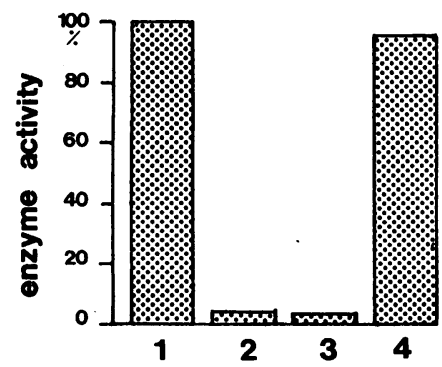

Figure 5. Relative enzyme activities of human prolidase in transfected human fibroblasts. Enzyme activity from normal fibroblasts (1), patient 1 fibroblasts (2), patient 1 fibroblasts transfected with pCAGGS (3), and patient 1 fibroblasts transfected with pEPD-W (4). The immune precipitated activities were calculated based on the amount of protein in the cell extracts and expressed by the percentages in the control fibroblasts.
The nucleotide substitution led to replacement of Asp with Asn, in association with a change in the secondary structure of the prolidase protein subunit (from $\alpha$-helix to $\beta$-sheet). Further analysis of the expression confirmed that this replacement was responsible for the defective enzyme activity, with no relation to polymorphism.

The plasmid pEPD-W constructed from the expression vector pCAGGS and cDNA insert of human prolidase could synthesize a polypeptide with prolidase activity in the transfected cells. This result clearly revealed the expression of the human prolidase with enzymatic activity in the NIH 3T3 cells, therefore demonstrating that the human prolidase cDNA we used (pPD-1) contained all of the sequence information required for expression of the intact prolidase gene product. The $56-\mathrm{kD}$ product achieved a proper structure and conformation of a homodimer and generated activity of the enzyme. On the other hand, the plasmid pEPD-M with the Asp to Asn substitution synthesized a polypeptide with a markedly decreased enzyme activity. These results were compatible with our data on fibroblasts (3) and confirmed that the homozygous substitution was responsible for the markedly decreased enzyme activity.

These two patients are products of nonconsanguineous matings and these two families had no apparent kinship. However, these families were from the Middle East area and the particular mutation demonstrated in this study seemed to have originated in this area.

Restoration of prolidase activity after transfection implies that the mutation does not affect the processing of wild-type prolidase. Transformed cell lines expressing human prolidase after gene transfer represent a new source of enzyme for biochemical characterization. Similarly, transformed cells containing mutant prolidase genes, cloned from affected individuals with prolidase deficiency, can be used to obtain the mutant prolidase enzyme.

As active prolidase can be expressed in mammalian cells using a proper clone, the possibility of replacement gene therapy for prolidase deficiency can be given serious consideration.

\section{Acknowledgments}

We thank Dr. J. Miyazaki (Kumamoto University) for the expression vector pCAGGS, Dr. M. Takiguchi (Kumamoto University) for providing synthetic oligonucleotides, Dr. Ch. M. Lapiére and Dr. S. K. Wadman for fibroblasts from the patients, Dr. C. Setoyama (Kumamoto University) for NIH 3T3 cells, Dr. J. Miyazaki and Dr. C. Setoyama for valuable discussions, $M$. Ohara for helpful comments, and M. Hayashi for secretarial services.

This work was supported in part by a Grant-in-Aid from The Ministry of Education, Japan, and The Ministry of Health and Welfare, Japan, a grant for pediatric research from The Ministry of Health and Welfare, Japan, and a grant for research from IBM Japan.

\section{References}

1. Phang, J. M., and C. R. Scriver. 1989. Disorders of proline and hydroxyproline metabolism. In The Metabolic Basis of Inherited Disease. C. R. Scriver, A. L. Beaudet, W. S. Sly, and D. Valle, editors. McGraw-Hill Inc., New York. 577-597.

2. Endo, F., K. Motohara, Y. Indo, and I. Matsuda. 1987. Immunochemical studies of human prolidase with monoclonal and polyclo- 
nal antibodies: absence of the subunit of prolidase in a patient with prolidase deficiency. Pediatr. Res. 22:627-633.

2a.Tanoue, A., F. Endo, and I. Matsuda. 1990. Structural organization of the gene for human prolidase (peptide D) and demonstration of a partial gene deletion in a patient with prolidase deficiency. J. Biol. Chem. In press.

3. Endo, F., A. Tanoue, A. Kitano, J. Arata, D. M. Danks, C. M. Lapiere, Y. Sei, S. K. Wadman, and I. Matsuda. 1990. Biochemical basis of prolidase deficiency. Polypeptide and RNA phenotypes and the relation to clinical phenotypes. J. Clin. Invest. 85:162-169.

4. Boright, A. P., C. R. Scriver, G. A. Lancaster, and F. Choy. 1989. Prolidase deficiency: biochemical classification of alleles. Am. J. Hum. Genet. 44:731-740.

5. Endo, F., A. Hata, Y. Indo, K. Motohara, and I. Matsuda. 1987. Immunochemical analyses of prolidase deficiency and molecular cloning of a cDNA for prolidase of human liver. J. Inherited Metab. Dis. 10:305-307.

6. Endo, F., A. Tanoue, H. Nakai, A. Hata, Y. Indo, K. Titani, and I. Matsuda. 1989. Primary structure and gene localization of human prolidase. J. Biol. Chem. 264:4476-4481.

7. Maniatis, T., E. F. Fritsch, and J. Sambrook. 1982. Molecular Cloning: A Laboratory Manual. Cold Spring Harbor Laboratory, Cold Spring Harbor, NY. 545 pp.

8. Newman, P. J., J. Gorski, G. C. White II, S. Gidwiz, C. J. Cretney, and R. H. Aster. 1988. Enzymatic amplification of plateletspecific messenger RNA using the polymerase chain reaction. J. Clin. Invest. 82:739-743.

9. Saiki, R. K., S. Scharf, F. Faloona, K. B. Mullis, G. T. Horn, H. A. Erlich, and N. Arnheim. 1985. Enzymatic amplification of $\beta$ globin genomic sequences and restriction site analysis for diagnosis of sickle cell anemia. Science (Wash. DC). 230:1350-1354.

10. Sanger, F., and A. R. Coulson. 1975. A rapid method for determining sequences in DNA by primed synthesis with DNA polymerase. J. Mol. Biol. 94:441-448.

11. Kunkel, L. M., K. D. Smith, S. H. Boyer, D. S. Borgaonkar,
S. S. Wachtel, O. J. Miller, W. R. Berg, H. W. Jones, Jr., and J. M. Rary. 1977. Analysis of human Y-chromosome-specific reiterated DNA in chromosome variants. Proc. Natl. Acad. Sci. USA. 74:12451249.

12. Miyazaki, J., S. Takaki, K. Araki, F. Tashiro, A. Tominaga, K. Takatsu, and K. Yamamura. 1989. Expression vector system based on the chicken $\beta$-actin promoter directs efficient production of interleukin-5. Gene (Amst.). 79:269-277.

13. Felgner, P. L., T. R. Gadek, M. Holm, R. Roman, H. W. Chan, M. Wenz, J. P. Northrop, G. M. Ringold, and M. Danielsen. 1987 Lipofection: a highly efficient, lipid-mediated DNA-transfection procedure. Proc. Natl. Acad. Sci. USA. 84:7413-7417.

14. Hall, C. V., P. E. Jacob, G. M. Ringold, and F. Lee. 1983. Expression and regulation of Escherichia coli lac $\mathrm{Z}$ gene fusions in mammalian cells. J. Mol. Appl. Genet. 2:101-109.

15. Herbomel, P., B. Bourachot, and M. Yaniv. 1984. Two distinct enhancers with different cell specificities coexist in the regulatory region of polyoma. Cell. 39:653-663.

16. Endo, F., and I. Matsuda. 1981. Screening method for prolidase deficiency. Hum. Genet. 56:349-351.

17. Saiki, R. K., D. H. Gelfand, S. Stoffel, S. J. Schare, R. Higuchi, G. T. Horn, K. B. Mullis, and H. A. Erlich. 1988. Primer-directed enzymatic amplification of DNA with a thermostable DNA polymerase. Science (Wash. DC). 239:487-491.

18. Lapiére, Ch. M., and B. Nusgens. 1969. Plaies cutanées torpides et trouble du metabolism du collagene. Arch. Belg. Dermatol. 25:353356.

19. Lombeck, I., U. Wendel, J. Versieck, L. Van Ballenberghe, H. J. Bremer, M. Duran, and S. K. Wadman. 1986. Increased manganese content and reduced arginase activity in erythrocytes of a patient with prolidase deficiency (iminodipeptiduria). Eur. J. Pediatr. 144:571-573.

20. Laemmli, U. K. 1970. Cleavage of structural proteins during the assembly of head of bacteriophage T4. Nature (Lond.). 227:680685. 